\title{
Revealing Exciton and Metal-Ligand Conduction Band Charge Transfer Absorption Spectra in Cu-Zn-In-S Nanocrystals
}

\author{
Brener R. C. Vale, Etienne Socie, Leticia R. C. Cunha, Andre F. V. Fonseca, Roberto Vaz, \\ Jefferson Bettini, Jacques-E. Moser,* and Marco A. Schiavon*
}

Cite This: J. Phys. Chem. C 2020, 124, 27858-27866

Read Online

\section{ACCESS | Lill Metrics \& More | 回 Article Recommendations ｜（） Supporting Information}

ABSTRACT: Copper indium sulfide quantum dots (QDs) have attracted substantial attention in recent years due to environmental issues and diverse applications. We report the synthesis and characterization of copper-zinc-indium-sulfide (CZIS) QDs and CZIS treated with excess $\mathrm{Zn}^{2+}$ at different temperatures, denoted here as CZIS/ZnS 100 and CZIS/ZnS 200. $\mathrm{Zn}^{2+}$ can diffuse into the lattice by an exchange-cation reaction, replacing $\mathrm{Cu}^{+}$and $\mathrm{In}^{3+}$. We employed transient absorption (TA) spectroscopy to study the role of $\mathrm{Zn}^{2+}$ in the lattice. The data were treated by global analysis, which yielded the decay-associated spectra (DAS). Through DAS and secondorder derivative absorption spectra, we could for the first time isolate the excitonic contribution from the metal-ligand conduction band charge transfer $\left(\mathrm{ML}_{\mathrm{CB}} \mathrm{CT}\right)$ absorption in the TA spectrum, finding the hole localization lifetime by its spectral and dynamical features. The hole localization lifetime increases from 0.3 to $1.7 \mathrm{ps}$ by increasing the $\mathrm{Zn}^{2+}$ concentration. We also

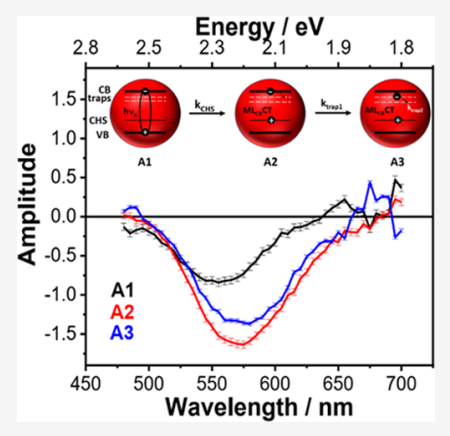
measured the electron-trapping constants to be dozens of picoseconds and nonradiative recombination larger than 1 ns. Finally, we concluded that suppression of the electron-trapping rate is not the only process responsible for increasing the photoluminescence quantum yield (PLQY); however, suppression of this process is important since it is the first step of the nonradiative recombination pathway. The detailed mechanism was proposed, and our results suggest that the introduction of $\mathrm{Zn}^{2+}$ in the lattice of copper indium sulfide (CIS) QDs could be beneficial for charge extraction.

\section{INTRODUCTION}

Heavy metal-free semiconductor nanocrystals have been extensively studied due to environmental issues. Ternary semiconductors such as copper indium sulfide (CIS) quantum dots (QDs) have displayed very interesting optoelectronic properties for applications in photovoltaic devices, ${ }^{1-4}$ lightemitting diodes (LEDs), ${ }^{5,6}$ photocatalytic hydrogen production, ${ }^{7}$ and biomarkers. ${ }^{8}$ CIS QD colloidal dispersions show long charge-carrier radiative recombination lifetimes (hundreds of nanoseconds), a large molar extinction coefficient from the visible to the near-infrared spectrum $\left(10^{5} \mathrm{~L} \mathrm{~mol}^{-1}\right.$ $\left.\mathrm{cm}^{-1}\right)$, and large Stokes shifts $(\sim 250-500 \mathrm{meV})$ free of reabsorption effects. 9,10

The CIS QDs are usually shelled with a wider-band-gap semiconductor, such as $\mathrm{ZnS}$ or $\mathrm{CdS}$, by similar experimental approaches used for $\mathrm{Cd}$ and $\mathrm{Pb}$ chalcogenide QDs. ${ }^{11-14}$ However, instead of a slight red shift in the absorption and emission spectra of the core-shell structure typically observed for type I core-shell alignments, a blue shift is observed in most cases. Some authors have ascribed the blue shift to alloying, cation-exchange, and etching processes. ${ }^{11,15-17}$ Cation-exchange occurs upon adsorption of Zn-ligand species on the CIS QD surface. Thus, an exchange reaction can take place when $\mathrm{Zn}^{2+}$ cations are incorporated in the $\mathrm{QD}$ and $\mathrm{Cu}^{+}$ or $\mathrm{In}^{3+}$ cations are extracted as M-ligand species. Alloying is slightly different from cation-exchange; the former occurs when $\mathrm{Zn}^{2+}$ from $\mathrm{ZnS}$ adsorbed on the surface diffuses inward, while $\mathrm{Cu}^{+}$or $\mathrm{In}^{3+}$ ions diffuse outward. Since both processes are quite similar, diverging only on the $\mathrm{Zn}^{2+}$ source, we interpreted the blue shift reported here by convention as a cation-exchange process. Cation-exchange with $\mathrm{Zn}^{2+}$ is also effective to increase the photoluminescence quantum yield (PLQY); for example, Lu et al. achieved with a record low fluence a two-photon amplified spontaneous emission (ASE) in Zn-processed CIS QDs. ${ }^{17}$ Etching occurs when species in the reaction medium promote the partial dissolution of the CIS QDs by extracting cations and/or $S^{2-}$ from the lattice, decreasing the particle size. According to Donega's group, the red shift occurs only for heteroepitaxial shell overgrowth. ${ }^{16}$ Moreover, they have demonstrated that the chemical route employed can control the different processes. Reactive precursors can promote the heteroepitaxial shell overgrowth, while nonreactive precursors promote cation-exchange or etching. ${ }^{16}$

Received: November 5, 2020

Revised: November 17, 2020

Published: December 2, 2020 
Many groups have devoted efforts to developing CIS QD optoelectronic properties, for example, to understand why the PL has a large Stokes shift, a broad emission band, and a long recombination lifetime. In the last 10 years, some proposals have been suggested to answer these questions, such as the forbiddances of optical transitions between the excited and ground states due to the tetragonal symmetry of the crystal lattice, ${ }^{18}$ exciton self-trapping process, ${ }^{19}$ and acceptor-donor pair states. ${ }^{20}$ However, the most accepted model suggested that the fluorescence is from the recombination between the electrons delocalized in the conduction band (CB) and the confined hole state ( $\mathrm{CHS}$ ) due to the trap related to copper ions. ${ }^{13,21,22}$ The same behavior has been observed for $\mathrm{Zn}$ and Cd chalcogenides intentionally doped with copper. ${ }^{19,23-25}$ Berends et al. proposed that the mechanism of electron-hole pair relaxation occurs in three steps, the first is hole localization on the CHS, which occurs in an ultrafast timescale less than $100 \mathrm{fs}$. However, they do not show any experimental evidence of hole localization, such as spectral or dynamical features. The second one is divided into two competing processes, which are electron trapping (recombination between the electron in the delocalized $\mathrm{CB}$ and electron traps) and radiative recombination between the electron in the delocalized $\mathrm{CB}$ and $\mathrm{CHS}$, and the third one is the nonradiative recombination between the trapped electron and the CHS. ${ }^{13}$

The Cu-related defect oxidation state can also play an important role in the electronic properties of CIS. The $\mathrm{Cu}^{+}$ defect is absorption-active due to the excitation of one of its $3 \mathrm{~d}$ electrons into the $\mathrm{CB}$ state, leaving the $\mathrm{Cu}$ cation in the $3 \mathrm{~d}^{9}$ configuration. $^{21,26,27}$ This transition, called metal-ligand (conduction band) charge transfer $\left(\mathrm{ML}_{\mathrm{CB}} \mathrm{CT}\right)$, leads to intragap absorption, which contributes to the apparent broadening of the absorption edge. On the other hand, $\mathrm{Cu}^{2+}$ is absorption-inactive, since the transition from the electron in the $3 \mathrm{~d}^{9}$ configuration cannot be transformed into the $3 \mathrm{~d}^{8}$ configuration by excitation of one electron into the $\mathrm{CB} .^{26}$ Therefore, $\mathrm{Cu}^{2+}$ defects do not contribute to the CIS absorption spectrum.

The composition also controls the Cu-related defect on CIS QDs. Because of charge compensating arguments, for stoichiometric samples with a 1:1 Cu-to-In ratio, the prevailing defects are an antisite pair of $\mathrm{Cu}^{+}$cations sitting on an $\mathrm{In}^{3+}$ lattice site with a double negative charge and an $\mathrm{In}^{3+}$ cation sitting on a $\mathrm{Cu}^{+}$lattice site with a double positive charge, as denoted in the Kröger-Vink notation of crystallographic defects represented as $\mathrm{Cu}_{\mathrm{In}}{ }^{\prime \prime}-\mathrm{In}_{\mathrm{Cu}} \cdots$. On the other hand, copper deficiency leads to $\mathrm{Cu}$ vacancies $\left(\mathrm{v}_{\mathrm{Cu}}{ }^{\prime}\right)$, which in turn facilitates the creation of charge compensating $\mathrm{Cu}^{2+}$ defects $\left(\mathrm{Cu}_{\mathrm{Cu}} \cdot\right)^{26}$

However, some questions are still unanswered in the literature: if the hole localizes at the CHS in an ultrafast timescale and the $\mathrm{ML}_{\mathrm{CB}} \mathrm{CT}$ transition has a lower probability transition than the excitonic one, is it possible to use transient absorption (TA) spectroscopy to experimentally measure the exciton absorption spectrum before hole localization and the free $\mathrm{ML}_{\mathrm{CB}} \mathrm{CT}$ transition? Why does the TA spectrum not overlap well with the second-order derivative absorption spectra? How can the excitonic and $\mathrm{ML}_{\mathrm{CB}} \mathrm{CT}$ transitions be influenced by $\mathrm{Zn}^{2+}$ incorporation? In this work, we report the synthesis and characterization of copper-zinc-indium-sulfide (CZIS) QDs and CZIS treated with excess $\mathrm{Zn}^{2+}$ at 100 and $200{ }^{\circ} \mathrm{C}$. We have employed ultrafast TA spectroscopy to answer the questions aforementioned.

\section{METHODS}

Chemicals and Materials. All chemicals were used as received without further purification. Indium chloride $\left(\mathrm{InCl}_{3}\right.$. $4 \mathrm{H}_{2} \mathrm{O}, 97 \%$ ), copper iodide (CuI, 99.5\%), n-dodecanethiol (DDT, 98\%), oleic acid (OA, 90\%), oleylamine (OAm, 70\%), 1-octadecene (ODE, 90\%), and acetone (99\%) were purchased from Sigma-Aldrich. Toluene (99\%) and zinc chloride $\left(\mathrm{ZnCl}_{2}, 97 \%\right)$ were purchased from Exodos Científica and Dinâmica Química, respectively.

Synthesis of CZIS QDs and Cation-Exchange Reaction. We prepared CZIS QDs by following a previous procedure described in the literature with slight modification. ${ }^{28}$ First, we prepared a solution containing all of the metals and the ligands. Briefly, we reacted $0.2 \mathrm{mmol}$ of $\mathrm{ZnCl}_{2}, 0.2 \mathrm{mmol}$ of $\mathrm{InCl}_{3}, 0.04 \mathrm{mmol}$ of $\mathrm{CuI}, 60 \mu \mathrm{L}$ of oleic acid, and $250 \mu \mathrm{L}$ of DDT dissolved in $8 \mathrm{~mL}$ of ODE in a $25 \mathrm{~mL}$ three-necked flask. The solution was heated to $90{ }^{\circ} \mathrm{C}$ under vacuum with magnetic stirring for $30 \mathrm{~min}$. Then, we heated the system under an argonium gas flow until $180{ }^{\circ} \mathrm{C}$ for $5 \mathrm{~min}$. Second, we reacted the metal solution with a sulfur solution. This step was performed by dropping the temperature to $160{ }^{\circ} \mathrm{C}$ and adding a mixture of $0.8 \mathrm{mmol}$ of $\mathrm{S}$ dispersed in $2 \mathrm{~mL}$ of OAm into the three-necked flask containing all of the metals and reacting for $10 \mathrm{~min}$ to allow for the growth of CZIS QDs.

To perform the cation-exchange reaction, we first prepared a $\mathrm{Zn}$ stock solution by dissolving $2.0 \mathrm{mmol}$ of $\mathrm{ZnCl}_{2}$ and $1 \mathrm{~mL}$ of OAm in $4 \mathrm{~mL}$ of ODE in a three-necked flask. The reaction mixture was heated to $90{ }^{\circ} \mathrm{C}$ under an argonium flow with magnetic stirring for $30 \mathrm{~min}$. Then, the flask was heated to 150 ${ }^{\circ} \mathrm{C}$ for $10 \mathrm{~min}$ to obtain a clear solution. Next, we cooled the flask to $50{ }^{\circ} \mathrm{C}$ for further use. For CZIS/ZnS 100 and CZIS/ ZnS 200 QDs, we used the CZIS QDs prepared as described above without the purification step. Then, we injected the $\mathrm{Zn}$ stock solution with a syringe into CZIS QDs at 100 and 200 ${ }^{\circ} \mathrm{C}$ for CZIS/ZnS 100 and CZIS/ZnS 200, respectively, for 30 min. For the purification step, we added acetone until the solution became turbid. Next, we centrifuged it at $7000 \mathrm{rpm}$ for $10 \mathrm{~min}$. Finally, we discarded the supernatant and dissolved the precipitate containing the QDs in $3 \mathrm{~mL}$ of toluene. We were able to prepare QDs that were stable for months by following this procedure.

Characterization. Steady-state fluorescence spectra were collected on a Horiba Jobin Yvon Fluorolog-3 fluorometer equipped with a Xe lamp of $450 \mathrm{~W}$. The excitation wavelength was set to $390 \mathrm{~nm}$. For time-correlated single-photon counting (TCSPC) analysis, we used a nano-LED as a pulsed source with a central wavelength at $390 \mathrm{~nm}$. We used a $1.00 \mathrm{MHz}$ repetition rate, with a resolution of 212 ps and an instrument response function (IRF) width of $1.3 \pm 0.1 \mathrm{~ns}$. Detection was performed at $90^{\circ}$ relative to the excitation source. Ultravioletvisible absorption spectra were acquired on a Perkin Elmer Lambda $950 \mathrm{UV} / \mathrm{Vis} / \mathrm{NIR}$ spectrometer. The fluorescence and absorption spectra in the steady state were acquired using cuvettes with an optical path of $10.00 \mathrm{~mm}$. For transient absorption spectroscopy, we used cuvettes with an optical path of $1.00 \mathrm{~mm}$.

Ultrafast transient absorption spectra of all samples were obtained using a fs chirped-pulse amplified (CPA) Ti:sapphire laser (Clark-MXR, CPA-2001). The pump beam at $390 \mathrm{~nm}$ was obtained by frequency-doubling the fundamental output of the laser $(778 \mathrm{~nm}, 120 \mathrm{fs}$ pulse duration, $1 \mathrm{kHz}$ repetition rate) in a $0.5 \mathrm{~mm}$ thick BBO crystal. The pump beam at $500 \mathrm{~nm}$ was 
obtained by directing the CPA output into a nonlinear parametric amplifier (NOPA), with subsequent compression to $70 \mathrm{fs}$ pulse duration with a pair of SF 10 prisms. The probe beam was produced by focusing a portion of the fundamental of the laser into an oscillating $\mathrm{CaF}_{2}$ crystal, yielding a white light continuum spanning from 400 to $780 \mathrm{~nm}$. The probe light used in all experiments had a smaller beam cross section and a weaker intensity compared to the pump beam so as to obtain a homogenous probed area. The pump beam was chopped at $500 \mathrm{~Hz}$. The dynamics of the photoinduced spectra were determined using a digitally controlled delay stage (PI) in the pump path. The polarization of the pump compared to the probe was set to the magic angle $\left(54.6^{\circ}\right)$ to consider only population dynamics. The probe beam was split before the sample into reference and signal branches, with the latter going through the sample, and both were separately sent into respective spectrographs (Princeton Instruments, Spectra Pro 2150i) and detected shot-to-shot by CCD cameras (Hamamatsu S07030-0906). The time resolution of the experiment was calculated to be 250 and 70 fs for pumping at 390 and 500 $\mathrm{nm}$, respectively.

\section{RESULTS AND DISCUSSION}

We prepared three different samples labeled here as CZIS, CZIS-ZnS 100, and CZIS-ZnS 200 according to a previous report in the literature with slight modifications (see the Methods section for details). ${ }^{28}$ Figure 1 a shows the PL and
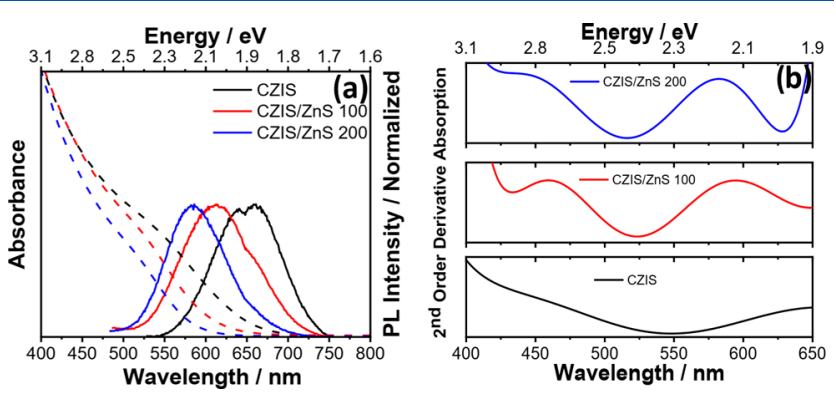

Figure 1. (a) Absorption (dashed line) and photoluminescence (solid line) spectra. (b) Second-order derivative of the absorption of CZIS, CZIS/ZnS 100, and CZIS/ZnS 200 QDs.

UV-Vis spectra (solid and dashed line, respectively) of the three prepared samples. The absorption spectra for all of the samples were normalized at $390 \mathrm{~nm}(3.2 \mathrm{eV})$ for their spectral shape comparison. The absorption band of the three samples is featureless, which is very common for CIS QDs. The featureless absorption spectrum is from the size and composition ( $\mathrm{Cu}: \mathrm{In})$ distribution in the ensemble. Furthermore, Gamelin's group has demonstrated that even NCs with identical sizes and compositions but with different $\mathrm{Cu}^{+}$and $\mathrm{In}^{3+}$ spatial positions can change the highest occupied molecular orbital (HOMO) and lowest unoccupied molecular orbital (LUMO) energy levels by as much as $1 \mathrm{eV}$, contributing to the inhomogeneous broadening of the spectroscopic properties. ${ }^{29}$ To measure the valence band (VB)-conduction band (CB) exciton band-edge $\left(h \nu_{x}\right)$ transition, we used the second-order derivative of the absorption spectrum (SODAS), a usual procedure used in the literature for featureless absorption spectra, as shown in Figure $1 \mathrm{~b}$. The calculated $h \nu_{x}$ transition through SODAS is summarized in Table S1 in the Supporting Information (SI). After $\mathrm{Zn}^{2+}$ incorporation, the temperature increases $h \nu_{x}$, and the PL spectra shift to higher energies. This behavior is typical for CIS QDs and is due to cation-exchange processes, in which $\mathrm{In}^{3+}$ and $\mathrm{Cu}^{+}$are replaced by $\mathrm{Zn}^{2+}$ in the lattice structure. The increase in the temperature facilitates the diffusion of $\mathrm{Zn}^{2+}$ into the lattice. Moser et al. conducted in situ PL measurements to control the cation-exchange reaction shell growth on CISe NCs. ${ }^{30}$ They concluded that the cation-exchange growth mechanism consists of a surface reaction of the $\mathrm{Zn}$ precursor, followed by the sequential solid-state diffusion of $\mathrm{Zn}^{2+}$ atoms into successive atomic monolayers. ${ }^{30}$ Density of state (DOS) studies explain the blue shift in the absorption and emission spectra: according to DOS, the $\mathrm{Cu}^{+}$and $\mathrm{S}^{2-}$ orbitals contribute to the VB-edge, while $\mathrm{In}^{3+}$ and $\mathrm{Cu}^{+}$contribute to the CB-edge for CIS QDs. When the $\mathrm{Zn}^{2+}$ concentration increases in the alloy (CZIS) QDs, $\mathrm{Zn}^{2+}$ (3d) orbitals also start to contribute to the CB-edge, which gradually shifts into higher energies. ${ }^{29}$ We also observed that the intragap absorption $\mathrm{ML}_{\mathrm{CB}} \mathrm{CT}$ band, with the absorption of approximately $600 \mathrm{~nm}(2.1 \mathrm{eV})$, progressively decreases from CZIS to CZIS/ZnS 200, and the explanation for this observation is discussed later with the TA results. The PL maximum, exciton transition energy, and the apparent Stokes shift are summarized in Table S1. We observed a substantial increase in the PLQY of the samples treated with $\mathrm{Zn}^{2+}$ at 100 and $200{ }^{\circ} \mathrm{C}$ compared to the PLQY of the CZIS sample. CZIS, CZIS/ZnS 100, and CZIS/ZnS 200 show a PLQY of 12,17 , and $54 \%$, respectively.

We carried out ultrafast TAS to measure the relaxation of the photoexcited species in CZIS QDs. The setup was previously described in another study. ${ }^{31} \mathrm{We}$ excited the samples with a pump pulse centered at $500 \mathrm{~nm}$ with pulse duration and a full width at half-maximum (FWHM) of approximately $70 \mathrm{fs}$ and probed them with a white light beam in the region between 400 and $780 \mathrm{~nm}$; see the SI for more details. The TA spectrum of the CIS QDs is interpreted as the
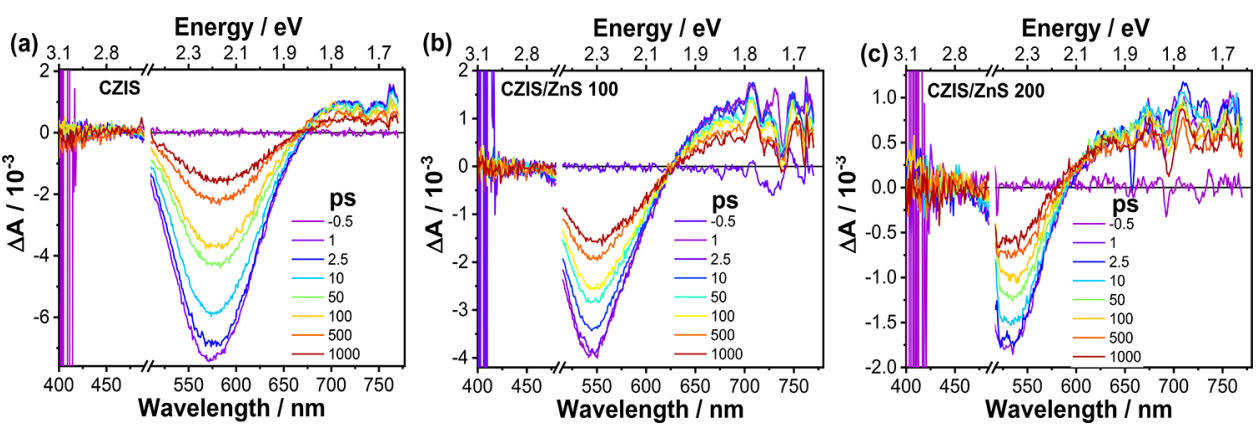

Figure 2. TA spectra showing different slices for (a) CZIS, (b) CZIS/ZnS 100, and (c) CZIS/ZnS 200 QDs. 

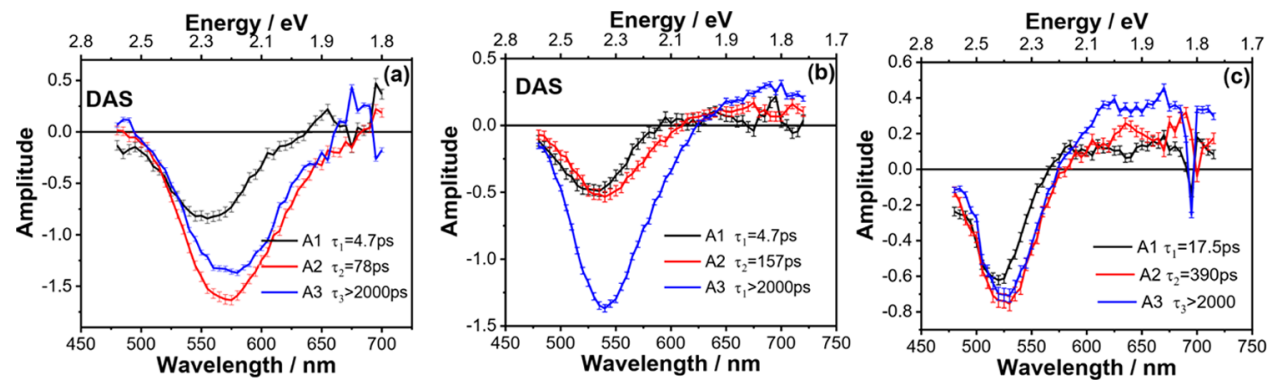

Figure 3. Decay-associated spectra (DAS) pumped at $390 \mathrm{~nm}$ for (a) CZIS, (b) CZIS/ZnS 100, and (c) CZIS/ZnS 200.

depopulation of electrons in the lowest-energy delocalized $\mathrm{CB}$ state; ${ }^{13}$ moreover, TAS measurements have shown that this state is 2 -fold degenerate. ${ }^{13,32}$ We measured the absorption cross section at $390 \mathrm{~nm}(3.2 \mathrm{eV})\left(\sigma_{3.2 \mathrm{eV}}\right)$, and we noticed that $\sigma_{3.2 \mathrm{eV}}$ did not change considerably from CZIS to CZIS-ZnS QDs treated at 100 or $200{ }^{\circ} \mathrm{C}$, as shown in Figure S1. We obtained absorption cross-section values close to the ones reported by Nagamine et al. ${ }^{33}$ Since $\sigma_{3.2 \mathrm{eV}}$ is proportional to the particle volume, the particle size did not change, excluding the contribution of the etching process to the blue shift in the emission and absorption spectra (see SI). ${ }^{33}$

Figure 2 shows the TA spectra of CZIS, CZIS/ZnS 100, and CZIS/ZnS 200 for different time delays, while Figure S3 shows full two-dimensional (2D) contour plots for the three samples. In these measurements, we used $\sim 7.7 \times 10^{14}$ photons $\mathrm{cm}^{-2}$, corresponding to an average of 0.3 excitons per particle. The average number of excitons per QD $\left(\left\langle\mathrm{N}_{x}\right\rangle\right)$ was calculated by the equation $\left\langle\mathrm{N}_{x}\right\rangle=j \sigma_{2.48 \mathrm{eV}}$, where $j$ is the density of photons and $\sigma_{2.48 \mathrm{eV}}$ is the absorption cross section at $2.48 \mathrm{eV}$; see the SI for details. The TA spectrum obtained at 1 ps for different photon fluences has the same shape in the range of $\sim 0.05-0.3$ $\left\langle\mathrm{N}_{x}\right\rangle$, suggesting that the effect of biexcitons on the spectral features is negligible, as shown in Figure S4. Moreover, the normalized bleach decay showed the same decay rate in this range as shown by photofluences, which means that the multiexcitonic processes are also negligible, as shown in Figure S5.

The TA spectrum is composed of ground-state bleach (GSB) and broad photoinduced absorption (PIA) bands, as reflected by the negative and positive peaks, respectively. We observed that the GSB is slightly red-shifted compared to the $h \nu_{x}$ measured by the SODAS for all of the systems studied here. Figure S6 shows the comparison of the TA, UV-Vis, and PL spectra. The GSB in semiconductor QDs is usually centered close to the ground-state absorption; even for CdSe QDs that are intentionally doped with copper ions, this red shift does not occur in the GSB. The red-shifted GSB for CIS QDs could be ascribed to the average of the two main processes, which are a VB-CB excitonic transition $\left(h \nu_{x \mathrm{Ta}_{\mathrm{a}}}\right)$ and intragap absorption due to the $\mathrm{ML}_{\mathrm{CB}} \mathrm{CT}$ transition $\left(h \nu_{\mathrm{Cu}}\right)$. Klimov's group recently carried out a study comparing two samples differing in copper contents but with similar size distributions. The SODAS for both samples are overlapped and have minimums located at $2.25 \mathrm{eV} .{ }^{26}$ The TA spectrum of the stoichiometric CIS QDs is different from that of the poor copper one in terms of shape and spectral energy. The deficient copper QDs showed two bands: a sharp one close to the intrinsic $\mathrm{VB}-\mathrm{CB}$ excitonic transition $\left(h \nu_{x, \mathrm{Ta}}\right)$ at $2.21 \mathrm{eV}$ (40 meV red-shifted compared to the $h \nu_{x}$ measured by the SODAS) and another located at $2.02 \mathrm{eV}$, which is ascribed to $\mathrm{h} \nu_{\mathrm{cu}}$. For the stoichiometric sample, just one broadband was centered at $2.04 \mathrm{eV}\left(210 \mathrm{meV}\right.$ red-shifted compared to $\left.h \nu_{x}\right)$, which is ascribed to $h \nu_{\mathrm{Cu}}$. In their study, they also noticed that the dynamics of the peak located at $2.21 \mathrm{eV}$ has a short-lived component with a time constant of $1.4 \mathrm{ps}$, which is absent for the stoichiometric sample. They attributed this short-lived component to hole localization. ${ }^{26}$ The GSB minimums for the samples studied here are summarized in Table S2. As seen from this table, the energy difference between the minimum of the GSB and the $h \nu_{x}$ for our samples is in the range of 97-63 $\mathrm{meV}$, decreasing from CZIS to CZIS/ZnS 200. These results are slightly greater than the energy difference between $h \nu_{x}$ and $h \nu_{x, \mathrm{TA}}(40 \mathrm{meV})$ and less than the energy difference between $h \nu_{x}$ and $h \nu_{\mathrm{Cu}}(210 \mathrm{meV})$ found by Fuhr et al. ${ }^{26}$

Since the GSB signal for CIS QDs is complex, we examined the GSB decay at different energies. Figure S7 shows the dynamics for different energies around the GSB for the three samples studied here. The dynamics were integrated into five nanometers around the selected probed energy to increase the signal-to-noise ratio. We selected energies as close as possible to $h \nu_{x}$ and at lower energies to detect the contribution of the $\mathrm{ML}_{\mathrm{CB}} \mathrm{CT}$ transition. The dynamics probed at different energies showed complex behaviors. For instance, we observed that the dynamics probed at a higher energy decay was faster than the dynamics at a lower energy for the CZIS QD sample. To obtain useful information from these data, we used global analysis to extract the dynamic and spectral behavior from the different samples; see the SI for details.

Figure 3 shows the decay-associated spectra (DAS) obtained from the global analysis pumped at $390 \mathrm{~nm}$. The DAS show the amplitude of a specific decay as a function of the corresponding wavelength. The difference between the DAS and the directly measured TA spectrum is that the former are calculated only based on the amplitude of the component decay, while the latter is based on the intensity of the signal, which depends on the concentration, oscillator strength, and decay. Thus, the low signal at a specific region in the experimental spectrum due to a low concentration or low oscillator strength can be hidden in a normal spectrum. Therefore, signals that are low but with steep decay are pronounced in DAS. The positive amplitude in DAS comes from a positive signal that decays over time or is due to a negative signal with an ultrafast timescale rise, while the negative amplitude only comes from negative signal decay. For all samples, we observed triexponential decay. We used a triexponential function plus an offset only for the samples excited at $500 \mathrm{~nm}$. All time constants for the different samples can be found in Tables S3 and S4. The different time constants observed when pumped at 390 or $500 \mathrm{~nm}$ are from the large difference between the pulse width durations for the pump 
pulses at $390(\sim 250 \mathrm{fs})$ and $500 \mathrm{~nm}(\sim 70 \mathrm{fs})$, and because of this, the intervals for data fitting are also different; see the SI for details. We observed that the GSB obtained from the DAS $\left(\mathrm{GSB}_{\mathrm{DAS}}\right)$ is shifted to a lower energy as the time constant increases from $A_{1}$ to $A_{3}$. For samples pumped at $500 \mathrm{~nm}$, we could not accurately measure the dynamics probed at approximately 500-510 $\mathrm{nm}$ for CZIS/ZnS 100 and CZIS/ $\mathrm{ZnS} 200$ QDs due to the strong scattering from the pump; see Figure S6. Because of this, the minimum of the $A_{1}$ component for these samples could not be seen in Figure S8. We can say that our results when pumped at $390 \mathrm{~nm}$ are rich in terms of spectral resolution but poor in terms of time resolution; however, the samples pumped at $500 \mathrm{~nm}$ are rich in terms of time resolution but poor in terms of spectral content.

Interestingly, we observed that the minimum of the $A_{1}$ component for all of the samples matches very well with the $h \nu_{x}$ measured by SODAS, and Table 1 summarizes all of these

Table 1. $h \nu_{x}$ and the "GSB" Energy Minimum Measured by SODAS and DAS Approaches, Respectively ${ }^{a}$

\begin{tabular}{lcccc}
\multicolumn{1}{c}{ samples } & $h \nu_{x} / \mathrm{eV} / \mathrm{nm}$ & $A_{1} / \mathrm{eV} / \mathrm{nm}$ & $A_{2} / \mathrm{eV} / \mathrm{nm}$ & $A_{3} / \mathrm{eV} / \mathrm{nm}$ \\
CZIS & $2.254 / 550$ & $2.234 / 555$ & $2.156 / 575$ & $2.138 / 580$ \\
CZIS/ZnS 100 & $2.371 / 523$ & $2.339 / 530$ & $2.296 / 540$ & $2.296 / 540$ \\
CZIS/ZnS 200 & $2.403 / 516$ & $2.384 / 520$ & $2.339 / 530$ & $2.339 / 530$
\end{tabular}

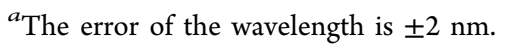

values. The DAS have been used for other semiconductors, and usually, the fastest component, denoted here as $A_{1}$, has been ascribed to cooling prior to relaxation of the band-edge. Moreover, the time constant related to cooling on the CIS QDs is on the order of hundreds of femtoseconds when pumped at $3.1 \mathrm{eV}$ or even slower ( $\sim 2 \mathrm{ps})$ under a high photofluence $\left(\left\langle\mathrm{N}_{x}\right\rangle \sim 4.0\right) .^{34,35}$ To check the contribution of cooling in our measurements, we probed the formation of the TA signal at an ultrafast timescale. This approach has been used for different materials and the rise time is related to the cooling process. ${ }^{36-38} \mathrm{We}$ fitted the data using an exponential growth equation convoluted with a Gaussian function, in which FWHM of the Gaussian function was fixed at 70 and $250 \mathrm{fs}$ to simulate the pulse width for pumping at 500 and 390 $\mathrm{nm}$, respectively. Figure 4a shows the growth dynamics for the CZIS, CZIS/ZnS 100, and CZIS/ZnS 200 QDs pumped at $390 \mathrm{~nm}$, while Figure 4b shows the dynamics for the CZIS sample pumped at $500 \mathrm{~nm}$. The data for CZIS/ZnS 100 and 200 pumped at $500 \mathrm{~nm}$ at a short timescale exhibited some artifacts due to the scattering from the pump. Since the excess energy is minimal for these samples (less than $0.08 \mathrm{eV}$ ), we did
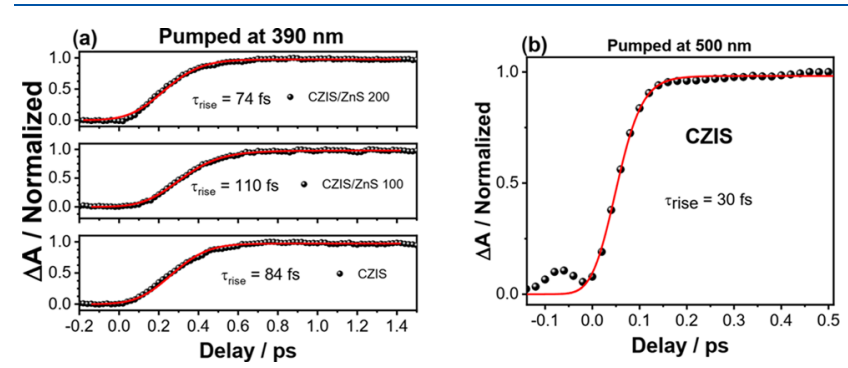

Figure 4. (a) GSB growth over time pumped at $390 \mathrm{~nm}$ and probed at 574,542 , and $530 \mathrm{~nm}$ for CZIS, CZIS/ZnS 100, and CZIS/ZnS 200 QDs, respectively. (b) GSB growth over time pumped at $500 \mathrm{~nm}$ and probed at $574 \mathrm{~nm}$ for CZIS QDs. not fit the data. As seen in Figure 4, the time constants found here were very short, indicating an instantaneous rise. Besides, we can exclude the possibility of cooling in this work because of two more reasons: first, the time constants found here were greater than 4 ps for all cases when excited at $390 \mathrm{~nm}$ and longer than $300 \mathrm{fs}$ when excited at $500 \mathrm{~nm}$ at a low fluence $\left(<\mathrm{N}_{x}>\sim 0.3\right)$ and second, when we applied excitation at 500 $\mathrm{nm}$, we did not expect cooling for CZIS/ZnS 200 because excitation is almost resonant. However, we can see an $A_{1}$ GSB blue-shifted compared to $A_{2}$ and $A_{3}$, which suggests that this signal is due to excitonic bleaching. In addition, if the signal in $A_{1}$ is from cooling, we expect a decrease in the time constant from CZIS to CZIS/ZnS 200 QDs because the extra energy decreases in this series, but the result is contrary to this. Thus, cooling is not the process observed here. Therefore, our results reported can be seen in the following way: after photoexcitation, the exciton is created and remains delocalized (i.e., the electron is delocalized into the $\mathrm{CB}$ and the hole is delocalized into the VB) for a specific time, which depends on the $\mathrm{Zn}$ concentration. This explains why the $\mathrm{GSB}_{\mathrm{DAS}}$ matches very well with the $h \nu_{x}$ measured by SODAS. Later, the hole is trapped in a CHS, shifting the $\mathrm{GSB}_{\mathrm{DAS}}$ minimum to a lower energy and broadening its shape.

Henceforth, we concentrate on the fastest time constant $\left(A_{1}\right.$ component). When we pumped the samples at $500 \mathrm{~nm}$, we noticed that the time constant of the $A_{1}$ component increased from CZIS to CZIS/ZnS 200 QDs, and the same is observed when pumped at $390 \mathrm{~nm}$, as shown in Tables S3 and S4, respectively. Since an increase in the time constant means a decrease in the probability of recombination, these results suggested that the hole localization rate decreases when more $\mathrm{Cu}^{+}$and $\mathrm{In}^{3+}$ are replaced by $\mathrm{Zn}^{2+}$ through the cation-exchange reaction. Klimov's group recently reported a hole localization of 1.4 ps for CIS QDs when pumped at $355 \mathrm{~nm} .{ }^{26}$ Donega's group suggested hole localization of fewer than 100 fs when pumped at $500 \mathrm{~nm} .{ }^{13}$ Note that the samples investigated in these studies are almost stoichiometric CIS QDs $\left(\left[\mathrm{Cu}^{+}\right] /\right.$ $\left.\left[\mathrm{In}^{3+}\right] \sim 1\right)$, indicating that this hole localization is faster than the process presented by our results, which makes sense with the trends presented here, since increasing the $\mathrm{Zn}^{2+}$ content increases the time constant. However, apart from Klimov's group, other studies have not shown experimental evidence of hole localization, such as spectral or dynamical features.

As we mentioned earlier, TA spectrum bleaching is composed of excitonic and $\mathrm{ML}_{\mathrm{CB}} \mathrm{CT}$ transitions. We also noticed that the FWHM of the TA spectrum decreases, and the shift in the GSB minimum in the DAS decreases from $A_{1}$ in CZIS to $A_{3}$ in CZIS/ZnS 200 (Table 2), which can be related to the suppression of the $\mathrm{ML}_{\mathrm{CB}} \mathrm{CT}$ transition owing to the decrease in the $\mathrm{Cu}^{+}$concentration. As we mentioned above, we noticed that the linear absorption spectra of CZIS/ZnS 100 and 200 QDs are sharper than those of CZIS QDs (Figure S2). We observed that the FWHM obtained from the TA spectra

Table 2. FWHM for $A_{1}$ and $A_{3}$ Components from the DAS and TA Spectrum

\begin{tabular}{lccc}
\multicolumn{1}{c}{ samples } & $A_{1} \mathrm{FWHM} / \mathrm{nm}$ & $A_{3} \mathrm{FWHM} / \mathrm{nm}$ & $\mathrm{TA}^{a} \mathrm{FWHM} / \mathrm{nm}$ \\
CZIS & 75 & 85 & 85 \\
CZIS/ZnS 100 & 67 & 73 & 70 \\
CZIS/ZnS 200 & 48 & 51 & 55
\end{tabular}

${ }^{a_{T}}$ The TA spectrum collected at 1 ps. 
does not change significantly over time (the result is not shown) due to the low sensitivity of low signals, as we explained earlier. Thus, the specific time is not important here, and we used the spectra at 1 ps because of the high signal-tonoise ratio. Therefore, the sharper absorption spectra and larger time constants found for CZIS/ZnS 100 and 200 suggested that $\mathrm{Zn}^{2+}$ decreases the concentration of $\mathrm{Cu}^{+}$and $\mathrm{In}^{3+}$, which decreases the contribution of $\mathrm{ML}_{\mathrm{CB}} \mathrm{CT}$ absorption to the absorption spectrum, decreasing the hole localization rate. It is worth mentioning that we cannot exclude the possibility of enhancement of the exciton binding energy with increasing $\mathrm{Zn}^{2+}$ concentration as the reason for a decreasing hole localization rate. However, this question is beyond the scope of this work, and a theoretical approach could be useful for this. Therefore, the longer hole localization lifetime with increasing $\mathrm{Zn}^{2+}$ could be easier for hole extraction since this process is more competitive with the hole localization.

According to the time constant and spectral signatures found for $A_{2}$ and $A_{3}$ obtained from DAS, we can say that $A_{2}$ and $A_{3}$ are related to electron trapping and nonradiative recombination, respectively. ${ }^{11,13,39}$ Nevertheless, due to the limitation of our delay line, we could measure the dynamics only until 1880 ps, and, thus, the time constant reported for the $A_{3}$ component is only an approximation that we observed for long-lived decay. We noticed that the electron-trapping process is much faster for CZIS than for CZIS/ZnS 100 or 200. This outcome suggests that $\mathrm{Zn}^{2+}$ incorporation is also effective in removing traps, making these materials interesting for applications such as LEDs and photovoltaic devices.

Finally, we carried out PL time-resolved measurements by a time-correlated single-photon counting (TCSPC) technique to measure the full relaxation process in the materials. The PL decay curves are shown in Figure S10. We monitored the PL decay at the maximum of the PL steady-state spectrum. The emission decay could be fitted to a biexponential function; Table S5 shows the time constants and respective amplitudes. The average PL lifetime was calculated by eq S7. According to the average PL lifetime and PLQY relationship, we could separate the radiative and nonradiative decay lifetimes. As seen from Figure S10, the PL decay curve did not change considerably upon $\mathrm{Zn}^{2+}$ cation-exchange process from CZIS to CZIS/ZnS 200. Since the PLQY increases from CZIS to CZIS-ZnS 200, we can say that the improvement in the PLQY is due to the increase in the radiative decay rate compared to that of the nonradiative one. We also probed the PL decay at different wavelengths, which is known as time-resolved emission spectra (TRES), for the three samples studied here. Figure $5 \mathrm{a}-\mathrm{c}$ shows the TRES of CZIS, CZIS/ZnS 100, and CZIS/ZnS 200 for different time delays. As can be seen, the dynamics probed at the blue edge of the emission spectrum decays faster compared to the dynamics probed at the red edge; the dynamic curves can be seen in Figure S11. As a result, the PL spectra red-shift as the time delay increases. We observed qualitatively that the red shift decreases as the $\mathrm{Zn}^{2+}$ content increases from CZIS to CZIS/ZnS 200. To quantify this red shift, we plotted the PL shift normalized at time $t=0$ for different time delays, $\Delta E=E(t)-E_{0}$, as shown in Figure $5 \mathrm{~d}$. The maximum PL shifts observed for CZIS QDs, CZIS/ $\mathrm{ZnS} 100$, and CZIS/ZnS 200 were $-155,-150$, and $-76 \mathrm{meV}$, respectively. The increase in $\mathrm{Zn}^{2+}$ content decreases the PL shift as a reduction of electron traps, in accordance with the TAS data. The same behavior was observed when CIS QDs were shelled with a thick layer of $\mathrm{ZnS}$ as a result of passivation
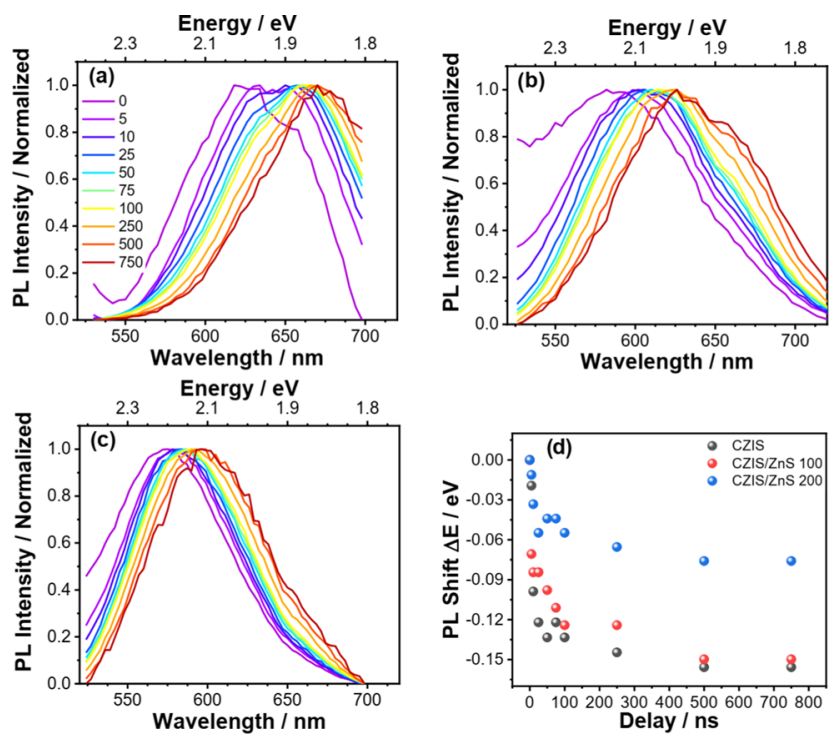

Figure 5. Time-resolved emission spectra of (a) CZIS, (b) CZIS/ZnS 100, (c) CZIS/ZnS 200, and (d) PL shift as a function of time delay.

of electron traps. ${ }^{40}$ As indicated by TRES results, suppression of electron traps can improve the emission band shape, obtaining a narrower PL spectrum, to produce saturated colors.

We have proposed the following mechanism for this work: since the probability of the $\mathrm{ML}_{\mathrm{CB}} \mathrm{CT}$ transition is much lower than that of the excitonic one, we can say that the first process to occur is excitonic recombination, considering the fact that cooling occurs faster than the time resolution of our experiment; additionally, the $\mathrm{GSB}_{\mathrm{DAS}}$ observed for $A_{1}$ accounted only for excitonic absorption, and the time constant is related to hole localization. Because of efficient hole localization, which relaxes the exciton and localizes the hole into the CHS located a few $\mathrm{meV}$ above the VB, the electron recombines with the hole at the CHS, and the $A_{2} \mathrm{GSB}_{\mathrm{DAS}}$ is red-shifted compared to $A_{1}$. The time constants related to $A_{2}$ and $A_{3}$ are related to the electron-trapping process and nonradiative recombination, respectively. The radiative recombination process takes a very long time, longer than 100 ns, as indicated by the PL decay curves, and do not contribute to the ultrafast TA spectrum. Radiative recombination is caused by an electron in the delocalized $\mathrm{CB}$ and a hole in the CHS, while nonradiative recombination is caused by the trapped electron and the hole in the CHS. We fitted our TA data with this physical model (see the SI for details), and we found time constants very close to the ones reported for the multiexponential fitting, Table 3. The fitted kinetic traces can be found in Figure S13. Scheme 1 shows a representation of the mechanism proposed in this work.

\section{CONCLUSIONS}

Therefore, we have shown that increasing the $\mathrm{Zn}^{2+}$ concentration in the lattice shifts absorption and PL to higher

Table 3. Time Constants Extracted from the TA Data Pumped at $500 \mathrm{~nm}$ Using the Physical Model

\begin{tabular}{lccl}
\multicolumn{1}{c}{ sample } & \multicolumn{1}{c}{$\tau_{\mathrm{CHS}} / \mathrm{ps}$} & $\tau_{\text {trap }} / \mathrm{ps}$ & \multicolumn{1}{c}{$\tau_{\text {nr }} / \mathrm{ps}$} \\
CZIS & $0.42 \pm 0.01$ & $9.46 \pm 0.1$ & $300 \pm 4$ \\
$\mathrm{CZIS} / \mathrm{ZnS} \mathrm{100}$ & $0.95 \pm 0.1$ & $23 \pm 1$ & $1183 \pm 60$ \\
$\mathrm{CZIS} / \mathrm{ZnS} \mathrm{200}$ & $2.82 \pm 0.2$ & $85 \pm 3$ & $>2000$
\end{tabular}


Scheme 1. Mechanism Proposed in This Work (See the Main Text)

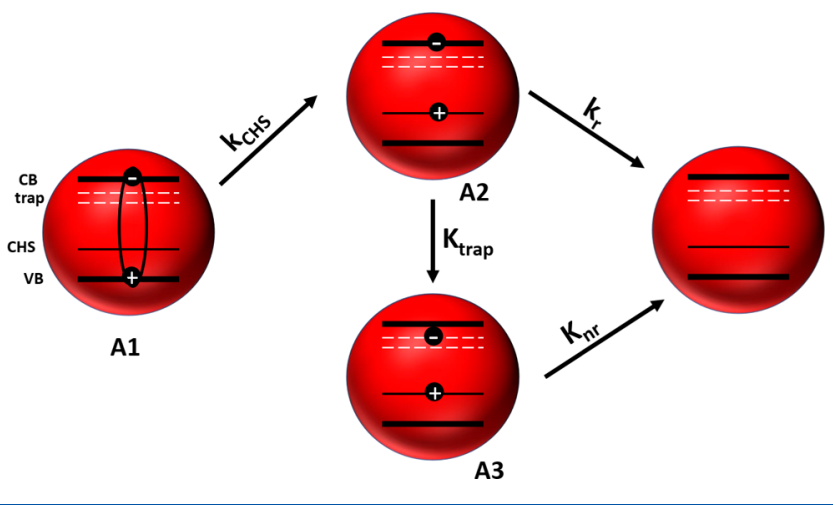

energies. The cation-exchange process is effective in increasing the PLQY of CIS QDs. Our methodology based on DAS obtained from TAS has spectrally and dynamically shown the exciton transition and hole localization, matching very well with the SODAS for all three samples studied here. In addition, we have shown that the time constant related to hole localization is dependent on the $\mathrm{Zn}^{2+}$ concentration, becoming slower for larger concentrations of $\mathrm{Zn}^{2+}$. The electron-trapping process decreased when CZIS QDs reacted with excess $\mathrm{Zn}^{2+}$. Note that suppression of the electron-trapping rate is not the only process responsible for increasing the PLQY; however, suppression of this process is important since it is the first step of the nonradiative recombination pathway. The TRPL studies in the nanosecond timescale agree well with the TA results and point out that reduction of electron traps reduces the PL inhomogeneity. Therefore, our results suggest that the introduction of $\mathrm{Zn}^{2+}$ in the lattice of CIS QDs could be interesting for LEDs and photovoltaic applications.

\section{ASSOCIATED CONTENT}

\section{SI Supporting Information}

The Supporting Information is available free of charge at https://pubs.acs.org/doi/10.1021/acs.jpcc.0c09681.

Global analysis; absorption cross-section measurements; PL maximum and second-order derivative absorption minimum; TA bleach minimum and true Stokes shift; dependence of TA bleaching on the photon fluence; estimated absorption cross-section wavelength dependence; TA spectra normalized at 1 ps and GSB decay at different photon fluences; decay curves probed at different energies; DAS pumped at $500 \mathrm{~nm}$; time constants for DAS pumped at 500 and $390 \mathrm{~nm}$; and PL decay curve obtained from TCSPC (PDF)

\section{AUTHOR INFORMATION}

\section{Corresponding Authors}

Jacques-E. Moser - Photochemical Dynamics Group, Institute of Chemical Sciences \& Engineering, École Polytechnique Fédérale de Lausanne, CH-1015 Lausanne, Switzerland; ○ orcid.org/0000-0003-0747-4666; Email: je.moser@ epfl.ch

Marco A. Schiavon - Grupo de Pesquisa Química de Materiais, Departamento de Ciências Naturais, Universidade Federal de São João Del-Rei, 36301-160 São João Del-Rei, Minas Gerais, Brazil; 이이.org/0000-0002-1553-5388; Email: schiavon@ufsj.edu.br

\section{Authors}

Brener R. C. Vale - Photochemical Dynamics Group, Institute of Chemical Sciences \& Engineering, École Polytechnique Fédérale de Lausanne, CH-1015 Lausanne, Switzerland; Grupo de Pesquisa Química de Materiais, Departamento de Ciências Naturais, Universidade Federal de São João Del-Rei, 36301-160 São João Del-Rei, Minas Gerais, Brazil

Etienne Socie - Photochemical Dynamics Group, Institute of Chemical Sciences \& Engineering, École Polytechnique Fédérale de Lausanne, CH-1015 Lausanne, Switzerland

Leticia R. C. Cunha - Grupo de Pesquisa Química de Materiais, Departamento de Ciências Naturais, Universidade Federal de São João Del-Rei, 36301-160 São João Del-Rei, Minas Gerais, Brazil

Andre F. V. Fonseca - Grupo de Pesquisa Química de Materiais, Departamento de Ciências Naturais, Universidade Federal de São João Del-Rei, 36301-160 São João Del-Rei, Minas Gerais, Brazil

Roberto Vaz - Grupo de Pesquisa Química de Materiais, Departamento de Ciências Naturais, Universidade Federal de São João Del-Rei, 36301-160 São João Del-Rei, Minas Gerais, Brazil

Jefferson Bettini - Laboratório Nacional de Nanotecnologia, Centro Nacional de Pesquisa em Energia e Materiais, 13083100 São Paulo, Brazil

Complete contact information is available at:

https://pubs.acs.org/10.1021/acs.jpcc.0c09681

\section{Author Contributions}

The manuscript was written through the contributions of all authors. All authors have given approval to the final version of the manuscript.

\section{Notes}

The authors declare no competing financial interest.

\section{ACKNOWLEDGMENTS}

B.R.C.V., E.S., and J.-E.M. are grateful to the Swiss National Science Foundation (SNF, grant no. 200021 175729) and the National Center of Competence in Research "Molecular Ultrafast Science and Technology" (NCCR-MUST), a research instrument of the SNF. B.R.C.V. thanks the Swiss Confederation through a Swiss Government Excellence Scholarship and the Federal University of São João del-Rei for the scholarship. M.A.S. acknowledges financial support from CAPES, CNPq, FINEP, and FAPEMIG.

\section{REFERENCES}

(1) Du, J.; Du, Z.; Hu, J. S.; Pan, Z.; Shen, Q.; Sun, J.; Long, D.; Dong, H.; Sun, L.; Zhong, X.; et al. Zn-Cu-In-Se Quantum Dot Solar Cells with a Certified Power Conversion Efficiency of $11.6 \%$. J. Am. Chem. Soc. 2016, 138, 4201-4209.

(2) Chiang, Y. H.; Lin, K. Y.; Chen, Y. H.; Waki, K.; Abate, M. A.; Jiang, J. C.; Chang, J. Y. Aqueous Solution-Processed offStoichiometric Cu-In-S QDs and Their Application in Quantum Dot-Sensitized Solar Cells. J. Mater. Chem. A 2018, 6, 9629-9641.

(3) Santos, C. I. L.; Machado, W. S.; Wegner, K. D.; Gontijo, L. A. P.; Bettini, J.; Schiavon, M. A.; Reiss, P.; Aldakov, D. Hydrothermal Synthesis of Aqueous-Soluble Copper Indium Sulfide Nanocrystals and Their Use in Quantum Dot Sensitized Solar Cells. Nanomaterials 2020, 10, 1-14.

(4) So, D.; Pradhan, S.; Konstantatos, G. Solid-State Colloidal $\mathrm{CuInS}_{2}$ Quantum Dot Solar Cells Enabled by Bulk Heterojunctions. Nanoscale 2016, 8, 16776-16785. 
(5) Boonsin, R.; Barros, A.; Donat, F.; Boyer, D.; Chadeyron, G.; Schneider, R.; Boutinaud, P.; Mahiou, R. Optical Properties and Reliability Studies of Gradient Alloyed Green Emitting $(\mathrm{CdSe})_{\mathrm{x}}(\mathrm{ZnS})_{1-\mathrm{x}}$ and Red Emitting $(\mathrm{CuInS} 2)_{\mathrm{x}}(\mathrm{ZnS})_{1-\mathrm{x}}$ Quantum Dots for White Light-Emitting Diodes. ACS Photonics 2018, 5, 462470.

(6) Chen, J.; Li, Y.; Wang, L.; Zhou, T.; Xie, R. J. Achieving DeepRed-to-near-Infrared Emissions in Sn-Doped Cu-In-S/ZnS Quantum Dots for Red-Enhanced White LEDs and near-Infrared LEDs. Nanoscale 2018, 10, 9788-9795.

(7) Sandroni, M.; Gueret, R.; Wegner, K. D.; Reiss, P.; Fortage, J.; Aldakov, D.; Collomb, M. N. Cadmium-Free $\mathrm{CuInS}_{2} / \mathrm{ZnS}$ Quantum Dots as Efficient and Robust Photosensitizers in Combination with a Molecular Catalyst for Visible Light-Driven $\mathrm{H}_{2}$ Production in Water. Energy Environ. Sci. 2018, 11, 1752-1761.

(8) Chetty, S. S.; Praneetha, S.; Vadivel Murugan, A.; Govarthanan, K.; Verma, R. S. Human Umbilical Cord Wharton's Jelly-Derived Mesenchymal Stem Cells Labeled with $\mathrm{Mn}^{2+}$ and $\mathrm{Gd}^{3+}$ Co-Doped $\mathrm{CuInS}_{2}$-ZnS Nanocrystals for Multimodality Imaging in a Tumor Mice Model. ACS Appl. Mater. Interfaces 2020, 12, 3415-3429.

(9) Berends, A. C.; Mangnus, M. J. J.; Xia, C.; Rabouw, F. T.; De Mello Donega, C. Optoelectronic Properties of Ternary I-III-VI Semiconductor Nanocrystals: Bright Prospects with Elusive Origins. J. Phys. Chem. Lett. 2019, 10, 1600-1616.

(10) Xia, C.; Wu, W.; Yu, T.; Xie, X.; Van Oversteeg, C.; Gerritsen, H. C.; De Mello Donega, C. Size-Dependent Band-Gap and Molar Absorption Coefficients of Colloidal $\mathrm{CuInS}_{2}$ Quantum Dots. ACS Nano 2018, 12, 8350-8361.

(11) Bose, R.; Ahmed, G. H.; Alarousu, E.; Parida, M. R.; Abdelhady, A. L.; Bakr, O. M.; Mohammed, O. F. Direct Femtosecond Observation of Charge Carrier Recombination in Ternary Semiconductor Nanocrystals: The Effect of Composition and Shelling. J. Phys. Chem. C 2015, 119, 3439-3446.

(12) Leach, A. D. P.; Macdonald, J. E. Optoelectronic Properties of $\mathrm{CuInS}_{2}$ Nanocrystals and Their Origin. J. Phys. Chem. Lett. 2016, 7, $572-583$.

(13) Berends, A. C.; Rabouw, F. T.; Spoor, F. C. M.; Bladt, E.; Grozema, F. C.; Houtepen, A. J.; Siebbeles, L. D. A.; De Mello Donegá, C. Radiative and Nonradiative Recombination in $\mathrm{CuInS}_{2}$ Nanocrystals and CuInS 2 -Based Core/Shell Nanocrystals. J. Phys. Chem. Lett. 2016, 7, 3503-3509.

(14) Reiss, P.; Protière, M.; Li, L. Core/Shell Semiconductor Nanocrystals. Small 2009, 5, 154-168.

(15) Zaiats, G.; Kinge, S.; Kamat, P. V. Origin of Dual Photoluminescence States in $\mathrm{ZnS}-\mathrm{CuInS}_{2}$ Alloy Nanostructures. J. Phys. Chem. C 2016, 120, 10641-10646.

(16) Berends, A. C.; Van Der Stam, W.; Hofmann, J. P.; Bladt, E.; Meeldijk, J. D.; Bals, S.; De Mello Donega, C. Interplay between Surface Chemistry, Precursor Reactivity, and Temperature Determines Outcome of $\mathrm{ZnS}$ Shelling Reactions on $\mathrm{CuInS}_{2}$ Nanocrystals. Chem. Mater. 2018, 30, 2400-2413.

(17) Lu, P.; Li, R.; Yao, N.; Dai, X.; Ye, Z.; Zheng, K.; Kong, W.; Fang, W.; Li, S.; Xu, Q.; et al. Enhancement of Two-Photon Fluorescence and Low Threshold Amplification of Spontaneous Emission of $\mathrm{Zn}$-Processed $\mathrm{CuInS}_{2}$ Quantum Dots. ACS Photonics 2018, 5, 1310-1317.

(18) Shabaev, A.; Mehl, M. J.; Efros, A. L. Energy Band Structure of $\mathrm{CuInS}_{2}$ and Optical Spectra of $\mathrm{CuInS}_{2}$ Nanocrystals. Phys. Rev. B 2015, 92, 1-9.

(19) Knowles, K. E.; Hartstein, K. H.; Kilburn, T. B.; Marchioro, A.; Nelson, H. D.; Whitham, P. J.; Gamelin, D. R. Luminescent Colloidal Semiconductor Nanocrystals Containing Copper: Synthesis, Photophysics, and Applications. Chem. Rev. 2016, 116, 10820-10851.

(20) Leach, A. D. P.; Macdonald, J. E. Optoelectronic Properties of $\mathrm{CuInS}_{2}$ Nanocrystals and Their Origin. J. Phys. Chem. Lett. 2016, 7, 572-583.

(21) Pinchetti, V.; Lorenzon, M.; McDaniel, H.; Lorenzi, R.; Meinardi, F.; Klimov, V. I.; Brovelli, S. Spectro-Electrochemical
Probing of Intrinsic and Extrinsic Processes in Exciton Recombination in I-III-VI 2 Nanocrystals. Nano Lett. 2017, 17, 4508-4517.

(22) Maiti, S.; Dana, J.; Jadhav, Y.; Debnath, T.; Haram, S. K.; Ghosh, H. N. Electrochemical Evaluation of Dopant Energetics and the Modulation of Ultrafast Carrier Dynamics in Cu-Doped CdSe Nanocrystals. J. Phys. Chem. C 2017, 121, 27233-27240.

(23) Knowles, K. E.; Nelson, H. D.; Kilburn, T. B.; Gamelin, D. R. Singlet-Triplet Splittings in the Luminescent Excited States of Colloidal $\mathrm{Cu}^{+}: \mathrm{CdSe}, \mathrm{Cu}^{+}: \mathrm{InP}$, and $\mathrm{CuInS}_{2}$ Nanocrystals: ChargeTransfer Configurations and Self-Trapped Excitons. J. Am. Chem. Soc. 2015, 137, 13138-13147.

(24) Yang, L.; Knowles, K. E.; Gopalan, A.; Hughes, K. E.; James, M. C.; Gamelin, D. R. One-Pot Synthesis of Monodisperse Colloidal Copper-Doped CdSe Nanocrystals Mediated by Ligand-Copper Interactions. Chem. Mater. 2016, 28, 7375-7384.

(25) Hughes, K. E.; Hartstein, K. H.; Gamelin, D. R. Photodoping and Transient Spectroscopies of Copper-Doped CdSe/CdS Nanocrystals. ACS Nano 2018, 12, 718-728.

(26) Fuhr, A.; Yun, H. J.; Crooker, S. A.; Klimov, V. I. Spectroscopic and Magneto-Optical Signatures of $\mathrm{Cu}^{1+}$ and $\mathrm{Cu}^{2+}$ Defects in Copper Indium Sulfide Quantum Dots. ACS Nano 2020, 14, 2212-2223.

(27) Fuhr, A.; Alexandrova, A.; Sautet, P. Stoichiometry-Controllable Optical Defects in $\mathrm{Cu}_{\mathrm{x}} \mathrm{In}_{2-\mathrm{x}} \mathrm{S}_{\mathrm{y}}$ Quantum Dots for Energy Harvesting. J. Mater. Chem. A 2020, 8, 12556-12565.

(28) Xiang, W.; Ma, X.; Luo, L.; Cai, W.; Xie, C.; Liang, X. Facile Synthesis and Characterization of Core/Shell Cu-In-Zn-S/ZnS Nanocrystals with High Luminescence. Mater. Chem. Phys. 2015, $149,437-444$

(29) Nelson, H. D.; Gamelin, D. R. Valence-Band Electronic Structures of $\mathrm{Cu}^{+}$-Doped $\mathrm{ZnS}$, Alloyed Cu-In-Zn-S, and Ternary $\mathrm{CuInS}_{2}$ Nanocrystals: A Unified Description of Photoluminescence across Compositions. J. Phys. Chem. C 2018, 122, 18124-18133.

(30) Moser, A.; Yarema, M.; Lin, W. M. M.; Yarema, O.; Yazdani, N.; Wood, V. In Situ Monitoring of Cation-Exchange Reaction Shell Growth on Nanocrystals. J. Phys. Chem. C 2017, 121, 24345-24351.

(31) Vale, B. R. C.; Socie, E.; Burgos-Caminal, A.; Bettini, J.; Schiavon, M. A.; Moser, J. E. Exciton, Biexciton, and Hot Exciton Dynamics in $\mathrm{CsPbBr}_{3}$ Colloidal Nanoplatelets. J. Phys. Chem. Lett. 2020, 11, 387-394.

(32) Li, L.; Pandey, A.; Werder, D. J.; Khanal, B. P.; Pietryga, J. M.; Klimov, V. I. Efficient Synthesis of Highly Luminescent Copper Indium Sulfide-Based Core/Shell Nanocrystals with Surprisingly Long-Lived Emission. J. Am. Chem. Soc. 2011, 133, 1176-1179.

(33) Nagamine, G.; Nunciaroni, H. B.; Mcdaniel, H.; Efros, A. L.; De Brito Cruz, C. H.; Padilha, L. A. Evidence of Band-Edge Hole Levels Inversion in Spherical CuInS 2 Quantum Dots. Nano Lett. 2018, $18,6353-6359$.

(34) Debnath, T.; Maiti, S.; Maity, P.; Ghosh, H. N. Subpicosecond Exciton Dynamics and Biexcitonic Feature in Colloidal CuInS Nanocrystals: Role of In-Cu Antisite Defects. J. Phys. Chem. Lett. 2015, 6, 3458-3465.

(35) Debnath, T.; Ghosh, H. N. Ternary Metal Chalcogenides: Into the Exciton and Biexciton Dynamics. J. Phys. Chem. Lett. 2019, 10, $6227-6238$.

(36) Richter, A. F.; Binder, M.; Bohn, B. J.; Grumbach, N.; Neyshtadt, S.; Urban, A. S.; Feldmann, J. Fast Electron and Slow Hole Relaxation in InP-Based Colloidal Quantum Dots. ACS Nano 2019, 13, 14408-14415.

(37) Li, Y.; Lai, R.; Luo, X.; Liu, X.; Ding, T.; Lu, X.; Wu, K. On the Absence of a Phonon Bottleneck in Strongly Confined $\mathrm{CsPbBr}_{3}$ Perovskite Nanocrystals. Chem. Sci. 2019, 10, 5983-5989.

(38) Chen, J.; Messing, M. E.; Zheng, K.; Pullerits, T. CationDependent Hot Carrier Cooling in Halide Perovskite Nanocrystals. J. Am. Chem. Soc. 2019, 141, 3532-3540.

(39) Guo, Y.; Yang, F.; Zheng, X.; Tang, J.; Zhong, H.; Yu, A.; Wang, J.; Zou, B. Direct Observation of Surface Polarons in Capped $\mathrm{CuInS}_{2}$ Quantum Dots by Ultrafast Pump-Probe Spectroscopies. J. Phys. Chem. Lett. 2019, 10, 5297-5301. 
(40) Sun, J.; Ikezawa, M.; Wang, X.; Jing, P.; Li, H.; Zhao, J.; Masumoto, Y. Photocarrier Recombination Dynamics in Ternary Chalcogenide $\mathrm{CuInS}_{2}$ Quantum Dots. Phys. Chem. Chem. Phys. 2015, 17, 11981-11989. 